\title{
CRACKLE DETECTION AND CLASSIFICATION BASED ON MATCHED WAVELET ANALYSIS
}

\author{
M. Du*\# F.H.Y. Chan* F.K. Lam* and J. Sun** \\ * Department of Electrical and Electronic Engineering, \\ The University of Hong Kong, Hong Kong. \\ ** Department of Electronic and Communication Engineering, \\ South China University of Technology, Guangzhou, China. 510641 \\ E-mail: mhdu@eee.hku.hk
}

\begin{abstract}
Crackles have an explosive pattern in time domain, with a rapid onset and short duration. The timing, repeatability, and shape of crackles are important parameters for diagnosis. Therefore, automatic detection of crackles and their classification have important clinical value. Since crackles have a general characteristic shape, it is obvious that wavelet analysis can be exploited to detect crackles and classify them. In this paper, we present a new method for crackle detection which is based on 'matched' wavelet transform. We first modeled crackles as a mathematical function. Then we designed a matched mother wavelet based on this model. Applying a soft-threshold to the results of the continuous wavelet transform to suppress noise further, the optimal scale can be obtained. Crackles can be detected based on the envelope of the signal at optimal scale, and can be classified based on energy distribution with scale. Theory, methods and experimental results are given in detail in this paper.
\end{abstract}

Key words: Lung Sound, Crackle, Wavelet Transform

\section{INTRODUCTION}

Crackles are heard over the lungs in a variety of diseases. They convey diagnostic information. Crackles have an explosive pattern in time domain, with a vapid onset and short duration. The timing of inspiratory crackles are an important parameter of lung sound.[1] Crackle counts and their distribution over the chest wall have been used as objective signs of lung disease.

There are two kinds of crackles. Fine crackles are associated with conspicuous high frequency component while coarse crackles are associated with low frequency components. The classification of crackles as fine and coarse has a major clinical significance. The former are associated with interstitial lung diseases, while the latter are more prevalent in obstructive airway diseases.
Therefore, it is important to detect crackles and classify them as fine and coarse automatically.

An adaptive nonlinear filter was used by Ono et al. to separate fine crackles from lung sound[2]. Predictive filter with adaptive threshold was also used by some of us to detect crackles.[3] Murphy et al. classified crackles by measuring some parameters of the waveform in time domain.[4][5]. Munakata et al. developed criteria for separating fine and coarse crackles.[6] But there is still no report on techniques for both detection and classification apart from the one proposed by us[7].

In this paper, we improve our technique in [7] and use 'matched' wavelet transform to detect and classify crackles. Since this technique can give an optimal response in translated domain, we will show that in this sense, it is an optimal method for crackle detection and classification.

\section{CRACKLE MORPHOLOGY}

Crackles have a general characteristic shape --- a sharp, sudden deflection that is usually followed by a wave, as shown in fig. 1 .

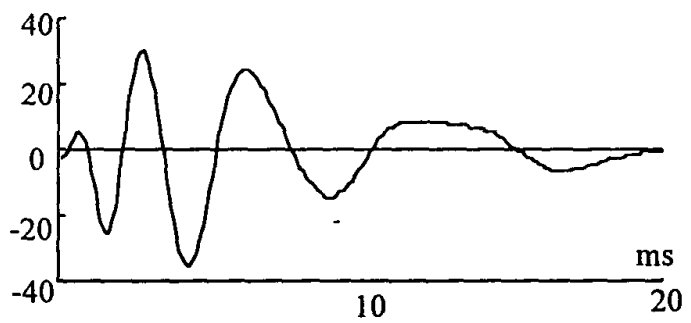

Fig .1 A typical crackle in time domain

When automatic detection is considered, details of crackle signal become important. Mori et al [8] performed a detailed analysis of crackles and found that crackles with an almost identical shape appeared in consecutive inspiration. The waveform had 4 to 10 positive and negative deflections, and the duration of each deflection was shortest initially and

\footnotetext{
"Visiting Scholar from Department of Electronic and Communication Engineering, South China University of Technology, Guangzhou, China.
} 
became progressively longer until a constant value was reached. A linearly progressive lengthening of zerocrossing intervals was also found. Based on these criteria, an idealized crackle can be constructed as:

$$
g(t)=\sin \left(2 \pi \cdot f_{0} \cdot t^{a_{1}}\right) \cdot t^{a_{2}} \cdot e^{1 / a_{3}}
$$

Where $a_{1}=0.5 ; a_{2}=1.49 ; a_{3}=0.78$ and $f_{0}=2.0$. A waveform of $\mathrm{g}(\mathrm{t})$ is shown in fig. 2 .

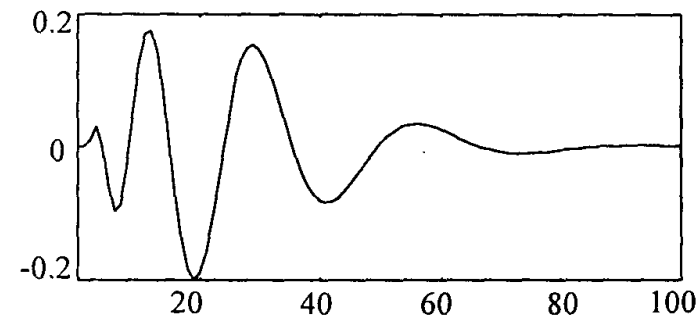

Fig 2. An ideal crackle

\section{WAVELET TRANSFORM}

The continuous wavelet transform of a signal $x(t)$ is defined by:[9-11]

$$
c w t_{x}(\tau, s)=\frac{1}{s} \int x(t) \psi\left(\frac{t-\tau}{s}\right) d t
$$

where $\psi(t)$ is the mother wavelet and $\frac{1}{s} \psi\left(\frac{t-\tau}{s}\right)$ the wavelet basis function. The parameter $s$ is called the scale, and $\tau$ is the translation of the wavelet function along the time axis. The mother wavelet $\psi(t)$ must have the following properties: (1) $\int \psi(t) d t=0$, i.e., they have zero DC component; (2) it is a bandpass signal; (3) it decays rapidly towards zero with time. Examples of wavelet are Morlet, Shannon, Haar, and second derivation of Gaussian, etc..[9]

Wavelet transform can be used to detect discontinuity in a signal[10], and hence can be exploited to detect crackles. Due to its multi-resolution property, wavelet transform can also be applied to crackle classification.

\section{MATCHED WAVELET}

Since wavelet transform (equation (2)) can be regarded as the computation of the cross-correlation between $x(t)$ and $\frac{1}{s} \psi\left(\frac{t}{s}\right)$, therefore, if the crackles model is used as the mother wavelet, the wavelet transform becomes an autocorrelation when $x(t)$ matches the scaled translated analysis function of (2). This will give an optimal response[12]. Hence it is able to localize crackles. As different optimal scale will correspond to different crackles, it is able to differentiate crackles.

Since the mean of $g(t)$ is less then $10^{-6}$ for scale 1 to 9 , which covers the frequency range of interest for lung sound, $g(t)$ can be regarded as meeting the admissibility condition, and can be used as a mother wavelet. In fact, the wavelet shown in fig. 2 has a scale of 3.

\section{CRACKLE DETECTION AND CLASSIFICATION CRITERIA}

\section{A. Matched wavelet analysis}

A segment of raw signal containing fine crackles is shown in fig.3(a). Using $g(t)$ as the mother wavelet, the wavelet transform of the signal can be obtained according to (2). Translated signal at several scales are showed in fig.3(b). It is clear that it has the maximum energy at scale about 2.5 . This means that wavelet of scale 2.5 is most similar to the fine crackles in this signal.

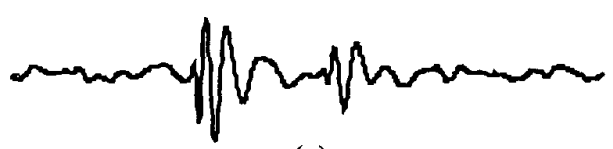

(a)

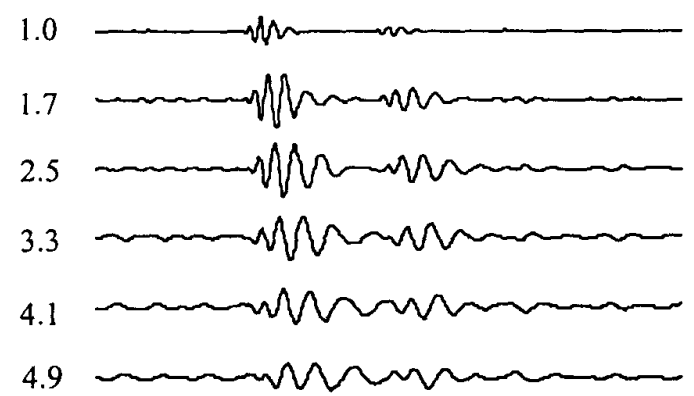

(b)

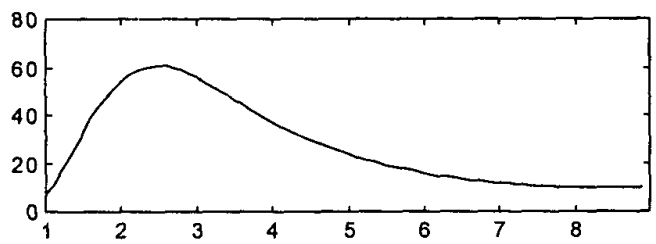

(c)

Fig.3 Matched wavelet analysis of a lung sound containing fine crackles. (a): Original; (b) WT at several scales; (c) energy distribution with scale. 
Fig.4(a). shows a segment of raw signal containing coarse crackles. When processed as described above, translated signal at several scales are obtained in fig.4(b). It is clear that it has the maximum energy at scale about 6.4 . This means that wavelet of scale 6.4 is most similar to coarse crackles in the signal.

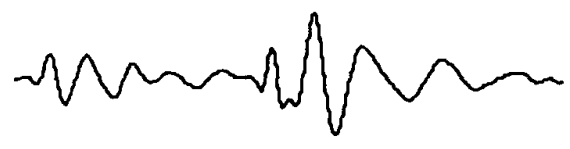

(a)

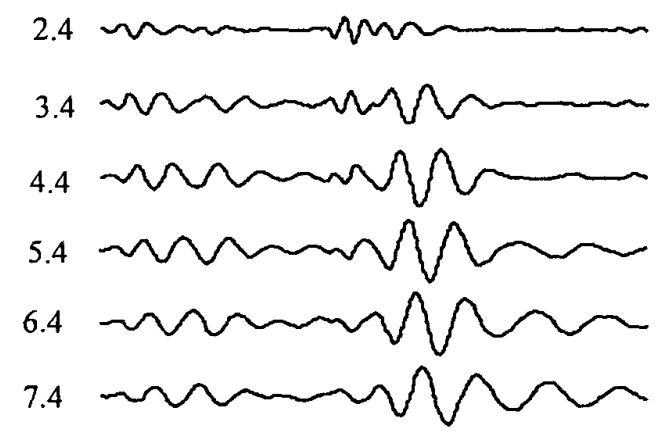

(b)

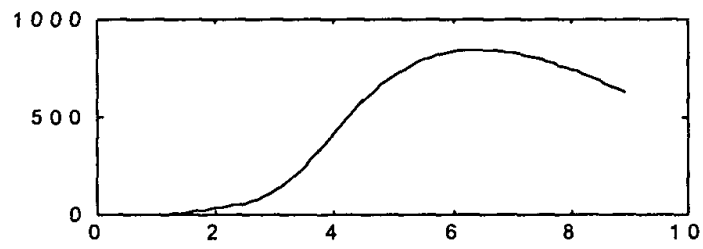

(c)

Fig.4 Matched wavelet analysis of a lung sound containing a coarse crackle. (a): Original; (b) WT at several scales;

(c) energy distribution with scale.

\section{B. Soft-threshold}

In order to further suppress noise, a soft-threshold is used as a nonlinear filter to eliminate noise in translated signals. The threshold at scale $s$ is defined by:

$$
\operatorname{thr}(s)=\log \left(1+c_{1} E(s)\right) \cdot e^{-s / c_{2}} \cdot c_{3}{ }^{s} \cdot c_{4}
$$

where $c_{1}=0.20, c_{2}=3.72, c_{3}=1.13$ and $c_{4}=4.10$.
The nonlinear filtering is carried out according to the following equation:

$$
c w t_{s, \tau}^{\prime}=\left\{\begin{array}{cc}
\left.\operatorname{sgn}\left(c w t_{s, \tau}\right)\left|c w t_{s, \tau}\right|-\operatorname{thr}(s)\right) & \text { if }\left|c w t_{s, \tau}\right| \geq \operatorname{thr}(s) \\
0 & \text { otherwise }
\end{array}\right.
$$

\section{Classification criteria}

From fig.3(b) and fig. 4(b), we can see that there exist an optimal scale at which the translated signals have the maximum energy. Statistical experiment is carried out and the probability distribution of optimal scale of fine and coarse crackles is shown in fig.5. It is clear that the optimal scales for fine crackles are between 2 to 3.2, while coarse crackles 3.3 to 7.4. Some coarse crackles have their optimal scales at 7.8. Therefore using this statistical chart, one can classify crackles as fine or coarse according to their optimal scale.

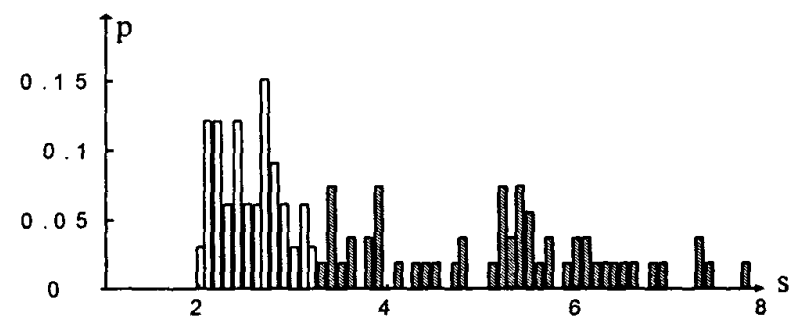

Fig. 5 Probability distribution of optimal scales blank: fine; shadowed: coarse

\section{CRACKLE DETECTION AND CLASSIFICATION}

\section{A. Signals and block diagram of processing}

Lung sounds are analyzed from a teaching tape by American College of Chest Physicians. The signals are digitized using DT2831 A/D converter at the sampling rate of $10 \mathrm{kHz}$. A moving overlapped window is applied to the original signal to select a segment to analyze. Wavelet transform is applied to this segment of signal. The optimal scale which has the maximum energy can be selected. According to the criteria discussed in section 5, crackles can be detected and classified The detection and classification block diagram is shown in fig.7

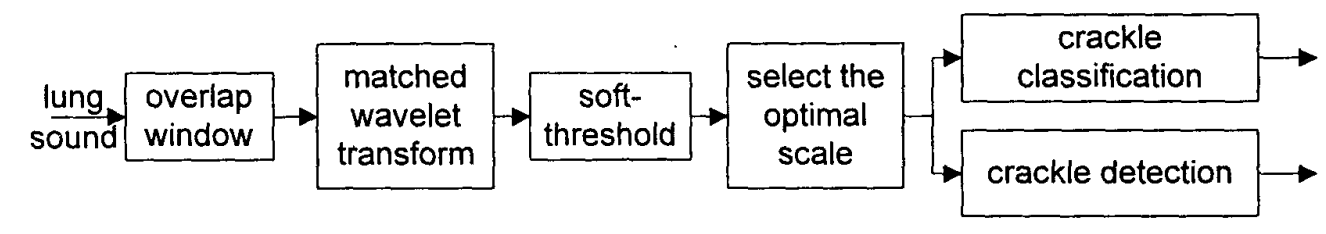

Fig.6 Block diagram for crackle detection and classification. 
Proceedings - 19th International Conference - IEEE/EMBS Oct. 30 - Nov. 2, 1997 Chicago, IL. USA

\section{B. Experimental results and discussions}

Fig. 7 and fig. 8 show two experimental results. We can see that not only large crackles can be located and classified, but also weak crackles. We have used 'timeexpanded' method[4] to verify our results.

Comparing to our previous works[7], this matched wavelet transform technique can give better results. The correct detection rate is about $99.8 \%$, while the correct classification rate is almost $100 \%$. As shown in [12] that matched wavelet transform can result in optimal response in translated signal, and hence can give an optimal envelop for crackle detection. Since a wavelet which has the same shape as crackles is used, the classification of crackles seems to be straight forward according to the optimal scale of translated signals. It is clear that this classification technique is equivalent to that of 'time-expanded' method. But the latter method works in time domain and is difficult to implement.
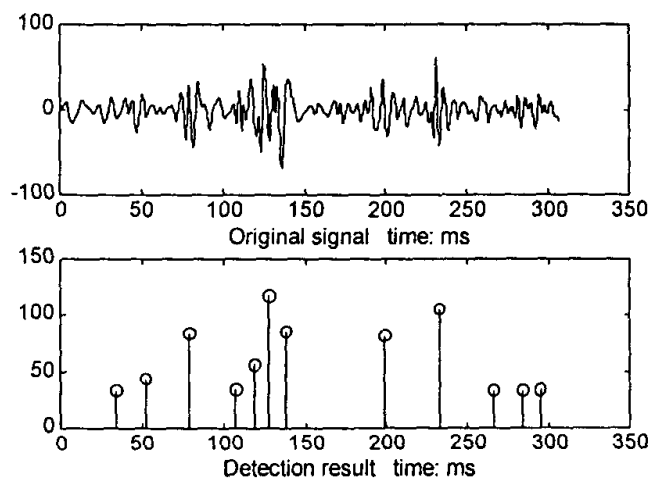

Fig.7 Detection and classification result of a segment of signal the teaching tape labeled as 'coarse crackles'. $o$ : coarse crackle
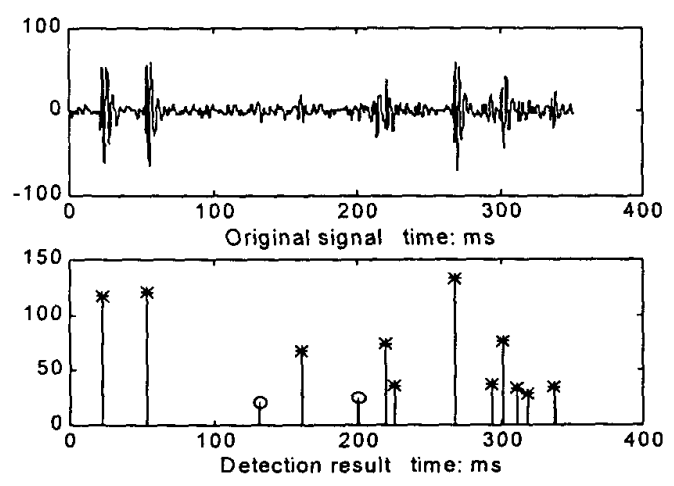

Fig.8 Detection and classification result of a segment of signal from the teaching tape labeled as 'fine crackles'.

$*$ : fine crackle; 0 : coarse crackle

\section{CONCLUSION}

Based on characteristics of crackles, we proposed a mathematical model for crackles. Using this model as a mother wavelet, matched wavelet transform can be carried out. Since matched wavelet transform can give optimal responses in translated domain, we can obtain optimal detection and classification results in this case. Experimental results show the success and efficiency of this technique.

\section{REFERENCES}

[1] N. Gavrily, D. W. Cugell, Breath Sounds Methodology. CRC Press, Inc. 1995

[2] M. Ono, K. Arakawa, M. Mori, et al. Separation of fine crackles from vesicular sounds by a nonlinear digital filter. IEEE Trans. Biomed. Eng. BME-36,286-291

[3] M. Du, J. Sun, Predictive filters applied to crackles detection. Proc. of China 13th Conference on Circuits and Systems. Chongqing, China, Oct. 1996. 152-155.

[4] R.L.H. Murphy, S.K. Holford, and W.C. Knowler, visual lung-sound characterization by time-expanded waveform analysis. New Eng. J. Med., 296, 968-971, 1978.

[5] J. Hoevers, and R.G. Loudon, Measuring crackles, Chest, 98,5, November 1990. 1240-1243

[6] M. Munakata, H. Ukita, I. Doi, et al, Spectral and waveform characteristic of fine and coarse crackles. Thorax, 46, 1991. 651-657

[7] M. Du, F.K. Lam, F.H.Y. Chan, J. Sun, Multiresolution decomposition applied to crackle detection. The 1997 IEEE International Conference on Systems, Man, and Cybernetics, Florida, USA. Oct.1997

[8] M. Mori, H. Morinari, et al. , Waveform and spectral analysis of crackles. Thorax, 35, 843-850, 1980.

[9] Y.T. Chan, Wavelet basics, Kluwer Academic Publishers, Boston, 1995.

[10]R, Y. Young, Wavelet theory and its applications, Kluwer Academic Publishers, 1993.

[11] S. Mallat, and W. L. Hwāng, Singularity detection and processing with wavelet, IEEE Trans. on Information Theory. Vol.38, No.2 March 1992.

[12]R. Priebe, and G. Wilson, Application of 'matched' wavelets to identification of metallic transients. Proc. of the IEEE-SP Inter. Symp. On Time-Frequency and Time-Scale Analysis. Oct.1992, Victoria, BC, Canada. 